\title{
Relationship-Based Top-K Concept Retrieval for Ontology Search
}

\author{
Anila Sahar Butt ${ }^{1,2}$, Armin Haller ${ }^{1,2}$, and Lexing Xie ${ }^{2}$ \\ 1 CSIRO, Canberra, Australia \\ 2 Australian National University \\ firstname.lastname@anu.edu.au
}

\begin{abstract}
With the recent growth of Linked Data on the Web there is an increased need for knowledge engineers to find ontologies to describe their data. Only limited work exists that addresses the problem of searching and ranking ontologies based on a given query term. In this paper we introduce DWRank, a two-staged bi-directional graph walk ranking algorithm for concepts in ontologies. We apply this algorithm on the task of searching and ranking concepts in ontologies and compare it with state-of-the-art ontology ranking models and traditional information retrieval algorithms such as PageRank and tf-idf. Our evaluation shows that DWRank significantly outperforms the best ranking models on a benchmark ontology collection for the majority of the sample queries defined in the benchmark.
\end{abstract}

\section{Introduction}

The growth in Linked Data coupled with the widespread use of ontologies in vertical domains (e.g. bioinformatics, e-commerce, internet-of-things etc.) highlights an increasing need to discover existing ontologies and the concepts and relations within. The benchmark ontology collection that we use in the evaluation of this paper, for example, includes 1022 ontologies that were retrieved through a Web crawl [2]. However, the potential to "reuse" these and other ontologies is hampered by the fact that it is hard to find the right ontology for a given use case. There are several established ontology libraries in vertical domains such as the Open Biological and Biomedical Ontologies library 1 or the BioPortal [14, where keyword queries are still the preferred method to find concepts and relations in the registered ontologies. However, since there may exist many ontologies that contain concepts and relations with their label matching the keyword query, the matches need to be usefully ranked. There has been some previous work, for example [7,14,13, to tackle the problem of finding and ranking ontologies. More recently, also dedicated ontology search engines have emerged [21], but the ranking algorithms they use are based only on document-ranking algorithms. Moreover, most of the ranking techniques in these ontology libraries and search engines only consider the popularity of terms in the ontology corpus, often using

${ }^{1}$ http://www .obofoundry.org/ 
the PageRank algorithm, which although effective in some cases 2, hinders the visibility of newly emerged well defined ontologies.

In this paper we propose a new ontology concept retrieval framework that uses a number of techniques to rate and rank each concept in an ontology based on how well it represents a given search term. The ranking in the framework is conducted in two phases. First, our offline ranking algorithm, DWRank, computes the centrality of a concept within an ontology based on its connectivity to other concepts within the ontology itself. Then, the authority of a concept is computed which depends on the number of relationships between ontologies and the weight of these relationships based on the authority of the source ontology. The assumption behind this is that ontologies that reuse and are reused by other ontologies are more authoritative than others. In a second, online query processing phase a candidate set for a top- $k$ concept is selected from the offline ranked list of ontologies and then filtered based on two strategies, the diverse results semantics and the intended type semantics. The resulting list of top- $k$ ranked concepts is then evaluated against a ground truth derived through a human evaluation published previously [2. Our evaluation shows that DWRank significantly outperforms the state-of-the-art ranking models on the task of ranking concepts in ontologies for all ten benchmark queries in the ontology collection.

The remainder of the paper is structured as follows. In Section 2 we describe the overall framework and briefly define some of the terms used throughout the paper. Section 3 describes the offline ranking phase of our framework, in particular the DWRank algorithm. Section 4 then describes the online query processing and filtering phase that is independent of the offline ranking model. We evaluate the DWRank algorithm with and without the additional filters in Section 5. We position our work in relation to state-of-the-art in Section 6] before we conclude in Section 7

\section{Relationship-Based Top-k Concept Retrieval}

In the following we first define the terms used throughout the paper. We then give a brief overview of the mechanics of the ranking framework.

\subsection{Preliminaries}

An ontology in this paper refers to a graph based formalisation $O=(V, E, L)$ of a domain knowledge. $V$ is a finite set of nodes where $v \in V$ denotes a domain concept in $O, E$ is the edge set where $\left(v, v^{\prime}\right) \in E$ denotes an explicit or implicit relationship between $v$ and $v^{\prime}$. $L$ is a labelling function which assigns a label $L(v)$ (resp. $L(e)$ or $L(O)$ ) to node $v$ (resp. an edge $e \in E$ or the ontology $O$ ). In practice the labelling function $L$ may specify (1) the node labels to relate the node to the referent concept, e.g. person, place and role; and (2) the edge labels as explicit relationships between concepts e.g., friendship, work and participation or implicit relationships e.g., sub-concept and super-concept, and (3) the ontology label to relate the ontology to the domain or some identity. 
Intra-Ontology Relationships. An intra-ontology relationship $I_{a}=\left(\left(v, v^{\prime}\right)\right.$, $O)$ is a directed edge $\left(v, v^{\prime}\right)$, where $\left(v, v^{\prime}\right) \in E(O)$ for $v \in V(O)$ and $v^{\prime} \in V(O)$.

Inter-Ontology Relationships. An inter-ontology relationship $I_{e}=\left(\left(v, v^{\prime}\right)\right.$, $\left.O, O^{\prime}\right)$ is a directed edge $\left(O, O^{\prime}\right)$, where $\left(v, v^{\prime}\right) \in \mathrm{E}(\mathrm{O}), L(v)=L(O), L\left(v^{\prime}\right)=$ $L\left(O^{\prime}\right)$ and $L\left(v, v^{\prime}\right)=$ owl:import 2 .

Forward Link Concepts. Forward link concepts $C_{F \operatorname{links}}(v, O)$ is a set of concepts $V^{\prime}$ in an ontology $O$, where $V^{\prime} \subset V(O)$ and $\forall v_{i} \in V^{\prime}, \exists\left(v, v_{i}\right) \in$ $E(O)$.

Back Link Concepts. Back link concepts $C_{B L i n k s}(v, O)$ is a set of concepts $V^{\prime \prime}$ in an ontology $O$, where $V^{\prime \prime} \subset V(O)$ and $\forall v_{j} \in V^{\prime \prime}, \exists\left(v_{j}, v\right) \in E(O)$.

\subsection{Overview of the Framework}

The framework is composed of two phases as shown in Fig. 1, The first phase is an offline phase where two indices, i.e. ConHubIdx and OntAuthIdx, are constructed for the whole ontology corpus. The second phase is an online query processing phase where a query is evaluated and the top- $k$ concepts are returned to the user.

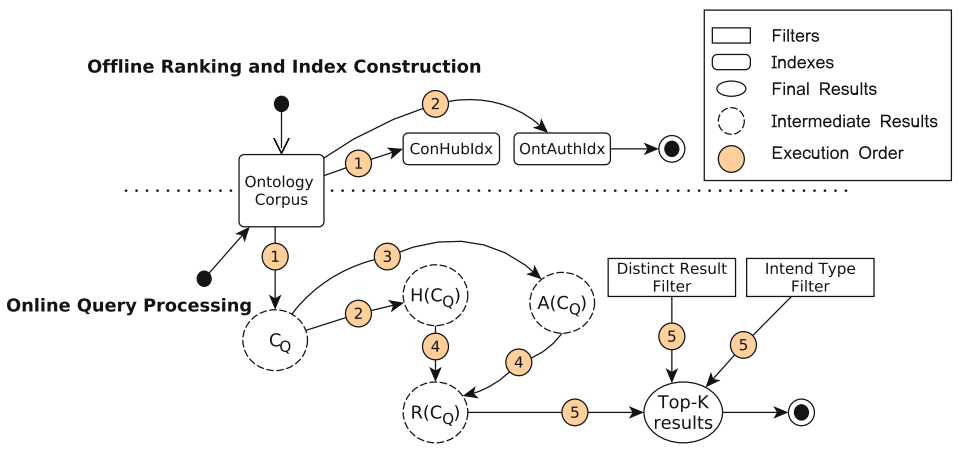

Fig. 1. Relationship-based top-k concept retrieval framework

Offline Ranking and Index Construction: The framework first constructs a ConHubIdx on all concepts and OntAuthIdx on all ontologies in the ontology corpus 0. The ConHubIdx maps each concept of an ontology to its corresponding hub score. Similarly, the OntAuthIdx maps each ontology to its precomputed authority score. The hub score and authority score are defined in Sec. 3.1

2 http://www .w3.org/2002/07/owl\#imports 
Online Query Processing: Upon receiving a query Q, the framework extracts the candidate result set $C_{Q}=\left\{\left(v_{1}, O_{1}\right), \ldots,\left(v_{i}, O_{j}\right)\right\}$ including all matches that are semantically similar to $\mathrm{Q}$ by querying the ontology repository. The hub score and authority score for all $(v, O) \in C_{Q}$ are extracted from the corresponding indices as $H\left(C_{Q}\right)$ and $A\left(C_{Q}\right)$ lists. A ranked list $R\left(C_{Q}\right)$ of a candidate result set is computed from $H\left(C_{Q}\right)$ and $A\left(C_{Q}\right)$ along with the text relevancy measure. $R\left(C_{Q}\right)$ is further filtered to satisfy two result set properties, i.e. the Diverse Result Semantics and the Intended Type Semantics, as introduced in Sec. 4.3.

\section{Offline Ranking and Index Construction}

In this section the offline ranking phase of the relationship-based top- $k$ concept retrieval framework is described (cf. Fig. 2). First, we introduce the ranking model in Section 3.1 and then we introduce the index construction based on the ranking model in Section 3.2 .

\subsection{DWRank: A Dual $W$ alk Based Ranking Model}

Our ranking model characterises two features of a concept to determine its rank in a corpus:

1. A concept is more important, if it is a central concept to the ontology within which it is defined.

2. A concept is more important, if it is defined in an authoritative ontology.

More precisely, first, the offline ranking module generates for each concept in the corpus a hub score, a measure of the centrality of a concept, i.e. the extent that the concept is related to the domain for which the ontology is formalised. Second, the authority score is generated as a measure of the authoritativeness of the ontology. A link analysis algorithm, i.e. PageRank, is performed that leverages the ontological structure and semantics to compute these scores. However, the difference between our model and a traditional PageRank-like algorithms is two-fold. Firstly, we perform the link analysis independently on each ontology to find a hub score and then only on the whole ontology corpus considering an ontology as a node and inter-ontology relationships as links. Secondly, we differentiate the type of relationship (i.e. inter-ontology and intra-ontology) and the direction of the walk varies on the basis of the type of the relationship. Our

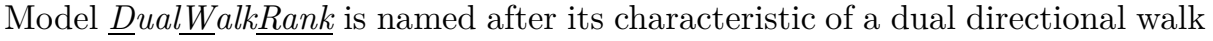
to compute the ranks of concepts.

HubScore: The Centrality of a Concept within an Ontology. The hub score is a measure of the centrality of a concept within an ontology. We define a hub function $\mathrm{h}(\mathrm{v}, 0)$ that calculates the hub score. The hub function is characterised by two features:

- Connectivity: A concept is more central to an ontology, if there are more intra-ontology relationships starting from the concept. 


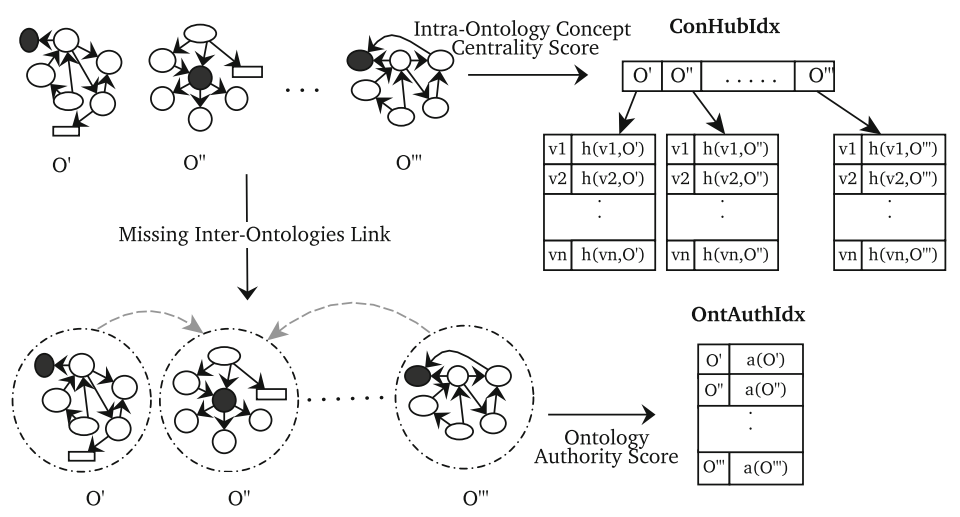

Fig. 2. Offline Ranking and Index Construction Phase

- Neighbourhood: A concept is more central to an ontology, if there is an intra-ontology relationship starting from the concept to another central concept.

According to these features, a concept accepts the centrality of another concept based on its forward link concepts (like a hub). The hub function is therefore a complete reverse of the PageRank algorithm [15] where a node accepts scores from its referent nodes i.e. back link concepts. We adopt a Reverse-PageRank [9] as the hub function to find the centrality of a concept within the ontology. The hub function is an iterative function and at any iteration $k$, the hub function is featured as Eq1]

$$
h_{k}(v, O)=\sum_{v_{i} \in C_{F L i n k s}(v, O)} \frac{h_{k-1}\left(v_{i}, O\right)}{\left|C_{B \text { Links }}\left(v_{i}, O\right)\right|}
$$

Within the original PageRank framework there are two types of links in a graph, strong and weak links. The links that actually exist in the graph are strong links. Weak links are artificially created links by a damping factor $\alpha$, and they connect all nodes to all other nodes. Since data-type relationships of a concept do not connect it to other concepts in an ontology, most PageRank-like algorithms adopted for ontology ranking consider only object type relationships of a concept while ignoring others. We adopt the notion of weak links in our hub function to be able to also consider data-type relationships along with object-type relationships for the ontology ranking. We generate a set of artificial concepts $V^{\prime}(O)$ in the ontology that act as a sink for every data-type relationship and label these concepts with the data type relationship label. i.e. $\forall v_{j} \in V^{\prime}, \mathrm{L}\left(v_{j}^{\prime}\right)=\mathrm{L}$ $\left(v_{i}, v_{j}^{\prime}\right)$. After incorporating weak links and weak nodes notions, Eq. 2 reflects the complete feature of our hub function. 


$$
h_{k}(v, O)=\frac{1-\alpha}{|V|}+\alpha \sum_{v_{i} \in C_{S F L i n k s}(v, O) \cup C_{W F \text { Links }}(v, O)} \frac{h_{k-1}\left(v_{i}, O\right)}{\left|C_{B \text { Links }}\left(v_{i}, O\right)\right|}
$$

In Eq. 2, $C_{S F L i n k s}(v, O)$ is a set of strong forward link concepts and $C_{W F L i n k s}(v, O)$ is a set of weak forward link concepts. Our hub function is similar to [22], but varies from it as we consider weak nodes and we are not considering relationships weights. The results presented in [22] also justify our choice of ReversePageRank over other algorithms to measure the centrality. We normalise the hub scores of each concept $v$ within an ontology $O$ through the $\mathbf{z}$-score of the concept's hub score after the last iteration of the hub function as follows:

$$
h_{n}(v, O)=\frac{h(v, O)-\mu_{h}(O)}{\sigma_{h}(O)}
$$

In Eq $3 h_{n}(v, O)$ is a normalised hub score of $v, \mu_{h}(O)$ is an average of hub scores of all concepts in the ontology and $\sigma_{h}(O)$ is the standard deviation of hub scores of the concepts in the ontology.

AuthorityScore: The Authoritativeness of a Concept. The authority score is the measure of the authoritativeness of a concept within an ontology. As mentioned earlier, the authoritativeness of a concept depends upon the authoritativeness of the ontology within which it is defined. Therefore, we define the authority function a (0) to measure the authority score of an ontology. Our authority function is characterised by the following two features:

- Reuse: An ontology is more authoritative, if there are more inter-ontology relationships ending at the ontology.

- Neighbourhood: An ontology is more authoritative, if there is an interontology relationship starting from an authoritative ontology to the ontology.

Based on these two features, an inter-ontology relationship $I_{e}\left(\left(v, v^{\prime}\right), O, O^{\prime}\right)$ is considered as a "positive vote" for the authoritativeness of ontology $O^{\prime}$ from $O$. The PageRank is adopted as the authority function, whereby each ontology is considered a node and inter-ontology relationships are considered links among

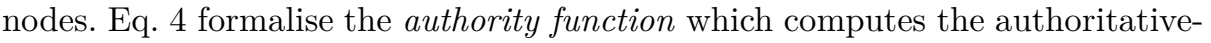
ness of $O$ at the $k$ th iteration.

$$
a_{k}(O)=\frac{1-\alpha}{|O|}+\alpha \sum_{O_{i} \in O_{B \text { Links }}(O)} \frac{a_{k-1}\left(O_{i}\right)}{\left|O_{F L i n k s}\left(O_{i}\right)\right|}
$$

In Eq. 4. $O_{B \text { Links }}(O)$ is a set of back link ontologies and $O_{F L i n k s}(O)$ is a set of forward link ontologies. The definition of $O_{F L i n k s}(O)\left(\operatorname{resp} . O_{B L i n k s}(O)\right)$ 
is similar to $C_{F L i n k s}(v, O)$ (resp. $\left.C_{B L i n k s}(v, O)\right)$, however, the links are interontology relationships.

Similar to the hub score, we also compute the $\mathbf{z}$-score of each ontology after the last iteration of authority function as follows:

$$
a_{n}(O)=\frac{a(O)-\mu_{a}(0)}{\sigma_{a}(0)}
$$

In Eq. 5. $a_{n}(O)$ is the normalised authority score of $v, \mu_{a}(0)$ is an average of the authority scores of all ontologies in the corpus and $\sigma_{a}(0)$ is the standard deviation of the authority scores of ontologies in 0.

DWRank Score. Finally, we define the DWRank $R_{(v, O)}$, as a function of the text relevancy, the normalised hub score and the normalised authority score. The function is described as a quantitative metric for the overall relevance between the query $\mathrm{Q}$ and the concept $v$; and the concept hub and authority score as follows:

$$
\begin{aligned}
R_{(v, O)} & =F_{V}(v, Q) *\left[w_{1} h(v, O)+w_{2} a(O)\right] \\
F_{V}(v, Q) & =\sum_{q \in Q} f_{s s}\left(q, \phi\left(q_{v}\right)\right)
\end{aligned}
$$

In Eq. 6] $w_{1}$ and $w_{2}$ are the weights for the hub function and the authority function. $F_{V}(v, \mathrm{Q})$ aggregates the contribution of all matched words of a node $v$, in an ontology $O$, to the query keywords $q \in \mathbf{Q}$. $f_{s s}$ returns a binary value : it returns 1 if $q$ has a match $\phi\left(q_{v}\right)$ in $v$, and 0 otherwise. The metric favours the nodes $v$ that are semantically matched to more keywords of the query $\mathbf{Q}$.

\subsection{Index Construction: An Execution of DWRank}

In this section, we explain the execution of the DWRank model and the construction of the indices.

ConHubIdx. A bi-level index where each entry in the index maps a concept of an ontology to its normalised hub score $h_{n}(v, O)$ as shown in Fig. 2 (top left). To construct the ConHubIdx for all ontologies in 0, (1) the hub function is executed in an iterative way to get the hub score of all the concepts in ontology $O$, and (2) after the last iteration, we compute the normalised hub scores and (3) insert the concepts along with their normalised hub scores in an ontology to the index.

OntAuthIdx. An index where each entry in the index maps an ontology to its normalised authority score $a_{n}(O)$ as shown in Fig. 2 (bottom left). To construct the OntAuthIdx on the corpus 0, (1) the authority function is executed to get an auth score of all the ontologies in 0, (2) after the last iteration, the normalised authority scores are computed, and (3) the ontology along with its normalised authority scores is inserted as an entry to the index. 
Inter-Ontology Relationships Extraction. As we mentioned earlier, the authority function leverages the inter-ontology relationships that are directed links among ontologies. If ontology OntA reuses the resources in ontology OntB, ontology OntA declares the reuse of resources through an OWL import property i.e. owl: imports. Since some ontology practitioners fail to explicitly declare the reuse of ontologies, the owl: imports relationships in an ontology are often inaccurate representations of the inter-ontology relationships. We therefore identify the implicit inter-ontology relationships by considering the reused resources in the corpus. Finding the implicit inter-ontology relationships involves the following steps:

1. Missing Relationships Detection: To find all missing inter-ontology relationships we identify the resources that appear in multiple ontologies. If a resource (referred to as "reused resource") is used in multiple ontologies (referred to as "hosting ontologies") then there must be some inter-ontology relationships. If these relationships are not explicitly defined then there are missing relationships among the ontologies.

2. Relationship Direction Identification: Since inter-ontology relationships are directed links between ontologies, another challenge is to find the direction of the missing relationships. A part of the ontology corpus in Fig. 2 (top right), contains a reused resource (i.e. filled node) that appears in three different ontologies $O^{\prime}, O^{\prime \prime}$ and $O^{\prime \prime \prime}$. In the absence of explicit relationships, some implicit relationships exist and to create these relationships we need to identify the direction of the relationships i.e. from $O^{\prime}$ to $O^{\prime \prime}$ and from $O^{\prime \prime \prime}$ to $O^{\prime \prime}$. To identify the direction, the namespace of the reused resource are used. If the namespace of the reused resource matches to the namespace of a hosting ontology (e.g. $\left.O^{\prime \prime}\right)$, then the ontology is selected as the "home ontology" of the reused resource and the inter-ontology relationships are directed from the hosting ontologies(i.e. $\left.O^{\prime}, O^{\prime \prime \prime}\right)$ to the home ontology i.e. $O^{\prime \prime}$.

3. Explicit Relationships Creation: Once the missing relationships and their directions are identified, we create explicit inter-ontology relationships using owl:imports properties.

An important point to consider is that although an ontology OntA may reuse more than one resource from another ontology $O n t B$ there will only be one inter-ontology relationship from OntA to OntB according to the semantics of the owl:imports property. Therefore, independently of the number of resources that are reused in $O n t A$ from $O n t B$, we create a single inter-ontology relationship from $O n t A$ to $O n t B$.

Table 1 and Table 2 show the top five ontologies in the benchmark ontology collection [2] and the corresponding number of inter-ontology relationships that are directed to these ontologies (i.e. reuse count) counted through explicit and implicit relationships, respectively. 
Table 1. Top five reused ontologies based on explicit inter-ontology relationships

\begin{tabular}{|l|l|}
\hline URI & Count \\
\hline \hline http://def.seegrid.csiro.au/isotc211/iso19150/-2/2012/basic & 36 \\
\hline http://purl.org/dc/elements/1.1/ & 25 \\
\hline http://www.ifomis.org/bfo/1.1 & 16 \\
\hline http://www.w3.org/2006/time & 16 \\
\hline http://www.ontologydesignpatterns.org/schemas/cpannotationschema.owl & 15 \\
\hline
\end{tabular}

Table 2. Top five reused ontologies based on implicit inter-ontology relationships

\begin{tabular}{|l|l|}
\hline URI & Count \\
\hline \hline http://www.w3.org/2002/07/owl\# & 881 \\
\hline http://www.w3.org/2000/01/rdf-schema & 361 \\
\hline http://www.w3.org/1999/02/22-rdf-syntax-ns & 298 \\
\hline http://xmlns.com/foaf/0.1/ & 228 \\
\hline http://www.w3.org/2004/02/skos/core & 140 \\
\hline
\end{tabular}

\section{Online Query Processing}

In this section, we first describe the concept retrieval task and then we outline the online query processing technique that finds the top-k ranked concepts for $\mathrm{Q}$ in 0 with the highest semantic relevance.

\subsection{Concept Retrieval Task.}

Given a query string $Q=\left\{q_{1}, q_{2}, \ldots, q_{k}\right\}$, an Ontology corpus $O=\left\{O_{1}, O_{2}, \ldots\right.$ . , $\left.O_{n}\right\}$ and a word sense similarity threshold $\theta$, the concept retrieval task is to find the $C_{Q}=\left\{\left(v_{1}, O_{1}\right), \ldots,\left(v_{i}, O_{j}\right)\right\}$ from 0 , such that there is a surjective function $f_{s j}$ from $\mathrm{Q}$ to $C_{Q}$ where (a) $v$ has a partial or an exact matched word $\phi\left(q_{v}\right)$ for $\mathrm{q} \in \mathrm{Q}(\mathrm{b})$ for a partially matched word, $\operatorname{Sen} \operatorname{Sim}\left(q, \phi\left(q_{v}\right)\right) \geq \theta$. We refer to $C_{Q}$ as a candidate set of $Q$ introduced by the mapping $f_{s j}$.

$\operatorname{Sen} \operatorname{Sim}\left(q, \phi\left(q_{v}\right)\right)$ is a word similarity measure of a query keyword and a partially matched word in $L(v)$.

\subsection{Query Evaluation}

In the online query evaluation (c.f Fig. 3), first a candidate set for a top- $k$ concept is selected from the ontology data store i.e. OntDataStore, and then the relevance of each concept is calculated based on the formulae defined in Eq. 6 . 


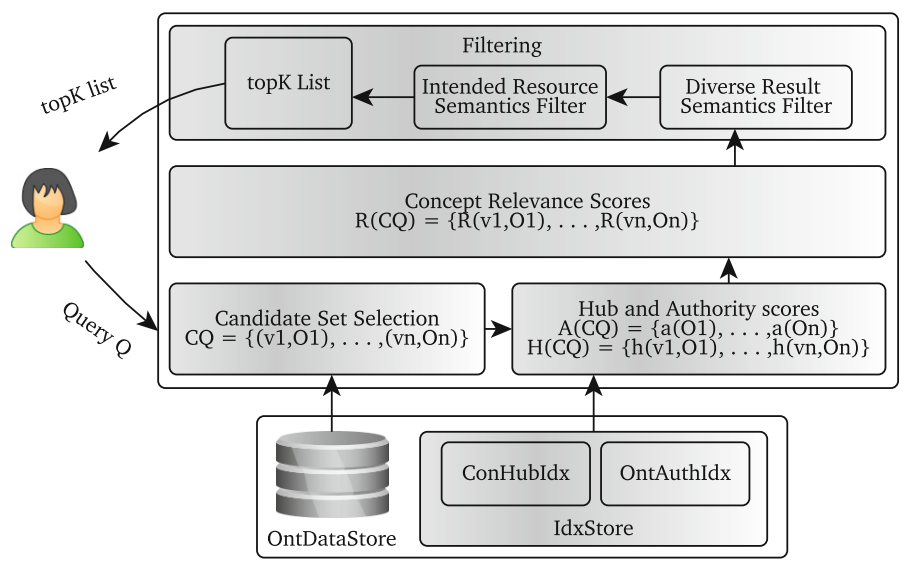

Fig. 3. Online Query Processing

Candidate Result Set Selection. A keyword query evaluation starts with the selection of a candidate set $C_{Q}$ for $\mathrm{Q}$. A candidate result set $C_{Q}$ is characterised by two features:

1. To be part of the candidate set a candidate concept $v$ must have at least one exact or partial match $\phi\left(q_{v}\right)$ for any query keyword $q \in \mathrm{Q}$ as part of the value of (a) rdfs:label (b) rdfs: comment (c) rdfs:description property; or $\exists$ $q \in \mathbf{Q} \mid \phi\left(q_{v}\right)$ is part of $L(v)$.

2. The word sense similarity of $q$ and $\phi\left(q_{v}\right)$ i.e. $\operatorname{senSim}\left(\mathrm{q}, \phi\left(q_{v}\right)\right)$ should be greater than the sense similarity threshold $\theta$.

In our current implementation, we check the word sense similarity using WordNet and set a word sense similarity threshold $\theta=0.85$. Each entry in a candidate list denotes a candidate concept ' $v$ ' and is a pair $(v, O)$ (shown in Fig. 3) of $v$ and $O$ where $v \in V(O)$. Since for the reused resources there are multiple hosting ontologies, therefore ' $v$ ' may have multiple entries in a candidate set if it is a reused resource.

Concept Relevance. For each entry in the candidate list, two scores are retrieved from the stored indices built during the offline ranking and index construction phase. The entry $(v, O)$ is used to retrieve the hub score of concept $v$ in ontology $O$ from the ConHubIdx, and the authority score of ontology $O$ from the OntAuthIdx. The two scores are combined according to the formulae of Eq. 6. that provides the final concept relevance of each $v$ to the Query $\mathbf{Q}$.

\subsection{Filtering Top-k Results}

In this section, we discuss the filtering strategies of our framework to enhance the semantic similarity of the results to the keyword query. We introduce two properties for the top- $k$ results: 
Diverse Results Semantic. Considering the semantics of a query allows us to remove repetitive results from the top-k results to increase the diversity in the result set. As mentioned earlier, if a candidate concept $v$ is reused/extended in ' $n$ ' hosted ontologies i.e. $\left\{O_{1}, O_{2}, \ldots, O_{n}\right\}$ then it may appear multiple times in a candidate result set (i.e. $\left.C_{Q}=\left\{\left(v, O_{1}\right),\left(v, O_{2}\right), \ldots,\left(v, O_{n}\right)\right\}\right)$. In this case we remove the duplicates from the candidate result set.

Intended Type Semantic. The semantic differentiates the intended type from the context resource of a concept. The label of a concept $v$ may have multiple keywords as a description of the concept e.g., the label of a concept in the GND ontology has the keywords "Name of the Person' 3 . Here "Name" is the intended type, whereas "Person" is the context resource. According to the intended type semantic property a concept should appear in the top- $k$ if and only if its intended type matches to at-least one of the query keywords $\mathrm{q} \in \mathbf{Q}$.

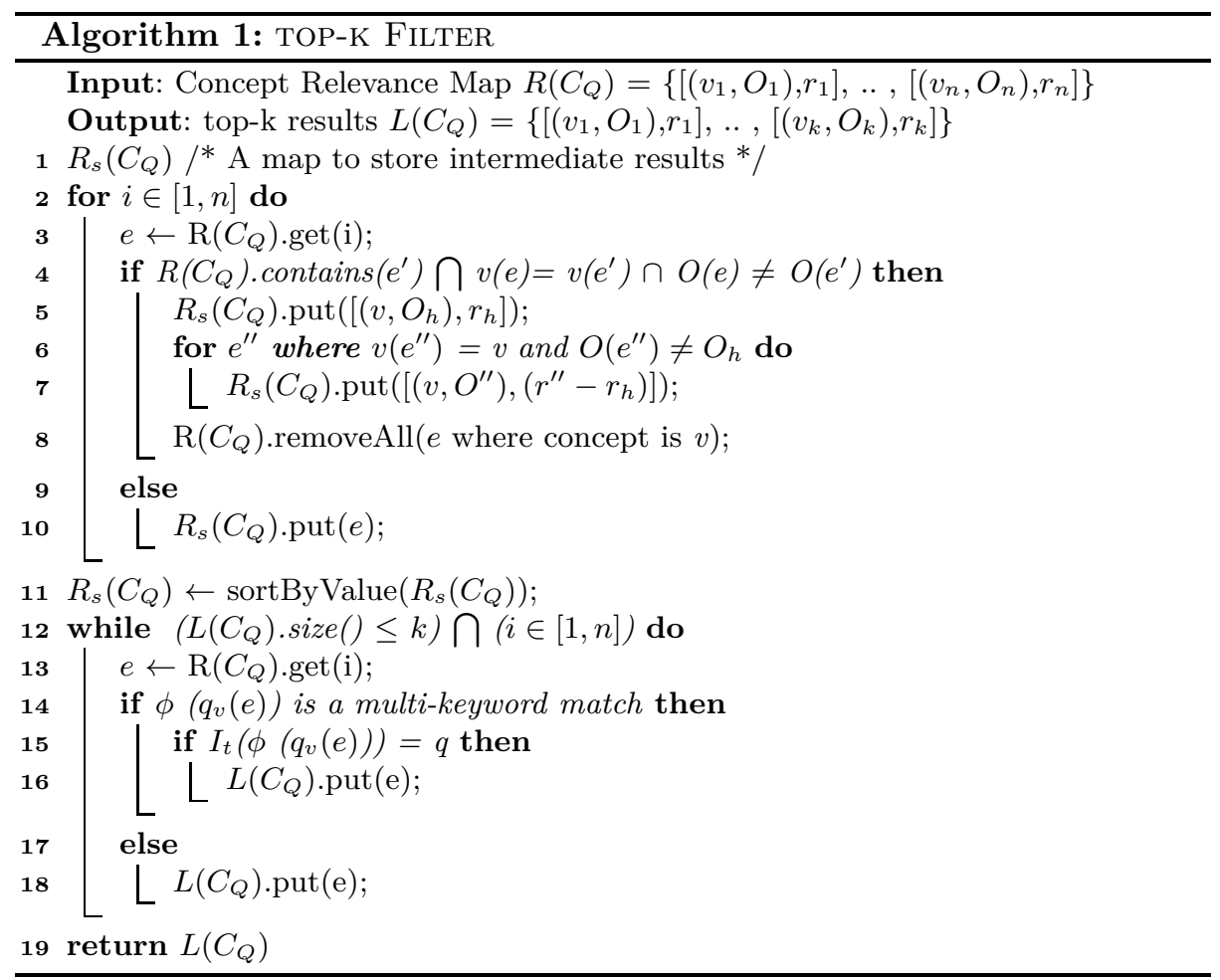

Algorithm 1 explains the top- $k$ results filtering process. It takes as input a Concept Relevance Map $R\left(C_{Q}\right)$ and returns the top- $k$ results. First, the diverse results semantics are preserved (line 2-10) for $R\left(C_{Q}\right)$, and then the check for

3 http://d-nb.info/standards/elementset/gnd\#Name0fThePerson 
the intended type semantics is applied (line 11-18) until the top- $k$ results are retrieved.

A map $R_{s}\left(C_{Q}\right)$ is initialised to store the intermediate results that preserve the diverse results semantics. All candidate concepts in $R\left(C_{Q}\right)$ that appear only once in $R\left(C_{Q}\right)$ preserve the diverse results semantics, therefore they become part of $R_{s}\left(C_{Q}\right)$ (line 10). For all reused concepts, first the home ontology $O_{h}(v)$ of the concept $v$ is identified. The entry $\mathrm{e}=[(v, O), r] \in R\left(C_{Q}\right)$ for which the ontology of the concept $v$ is its home ontology (i.e. $\mathrm{O}=O_{h}(v)$ ) becomes part of the $R_{s}\left(C_{Q}\right)$ (line 5). For all other entries $e^{\prime \prime}$ for $v$ a new entry is created by subtracting the relevance score of $e$ i.e. $r_{h}$ from the $r^{\prime \prime}$ and add it to the $R_{s}\left(C_{Q}\right)$ (line 6-7). The process decreases the relevance score of duplicate entries by a factor of $r_{h}$. Then all such $e^{\prime \prime}$ from $R\left(C_{Q}\right)$ are removed since they have already been dealt with through candidate concepts of $v$.

The next step is to check the intended type semantic. For brevity, a detailed discussion of the intended type checking is exempted from Algorithm 1 The ontology structure and the Information Retrieval method are used to identify the intended type. For a concept $v$, its sub-classes, super-classes and inter-ontology relationships are extracted as the context of $v$. The WS4. 4 API is used to calculate the similarity of different words in the concept $v$ with its context. The word that has a higher similarity score in regards to the context is considered as the intended type of the concept. However, to reduce the cost of ensuring the intended type semantic for top- $k$ results, the filter is only applied until we retrieved the top- $k$ results in the final results $L$. For this, first the $R_{s}\left(C_{Q}\right)$ is sorted in a decreasing order based on its relevance score $r$, so the more relevant results for query $\mathrm{Q}$ are at the top of the $R_{s}\left(C_{Q}\right)$ (line 11). Then the intended type of the candidate concept is checked only until ' $\mathrm{k}$ ' concepts are selected from $R_{s}\left(C_{Q}\right)$ or there are no more results in $R\left(C_{Q}\right)$ (line 12 ). If the concept $v$ has a single exact or partial matched word $\phi q_{v}(e)$ then by default it preserves the semantics and becomes part of $L\left(C_{Q}\right)$ (line 18), otherwise we check its intended type. If its intended type is equal to the query keyword $q \in \mathrm{Q}$, the concept is included in $L\left(C_{Q}\right)$ otherwise, it is ignored.

\section{Experimental Evaluation}

In the following we present an experimental evaluation of our relationship based top- $k$ concept retrieval framework on a benchmark suite CBRBench - Canberra Ontology Ranking Benchmark [2]. We conducted two sets of experiments to evaluate: (1) the effectiveness of the DWRank ranking model presented in Sec. 3 and (2) the effectiveness of the additional filtering phase presented in Sec. 4 .

\subsection{Experimental Settings}

To evaluate our approach we use a benchmark suite CBRBench [2], that includes a collection of ontologies, a set of benchmark queries and a ground truth established by human experts. This collection is composed of 1022 ontologies and

\footnotetext{
${ }^{4}$ https://code.google.com/p/ws4j/
} 
ten keyword queries: Person, Name, Event, Title, Location, Address, Music, Organization, Author and Time. The benchmark evaluates eight state-of-theart ranking algorithms: Tf-Idf[17], BM25[16], Vector Space Model (VSM) 18, Class Match Measure (CMM) [1], PageRank (PR) [15], Density Measure (DEM) [1, Semantic Similarity Measure (SSM) [1] and Betweenness Measure (BM) [1] on the task of ranking ontologies. We use the performance of these ranking models as the baseline to evaluate our approach. For a fair analysis, we implemented two versions of our approach: (1) DWRank: the DWRank model with the diverse root semantics (2) DWRank+Filter: the DWRank model with both the diverse root semantics and the intended type semantics. The reasoning for two different implementations of top-k concept retrieval framework is, we want to compare the effectiveness of DWRank model with the baseline ranking models and since CBRBench provides distinct top-k results of the baseline algorithms - which means diverse root semantics is considered while evaluating the baseline ranking models - therefore the DWRank model along with diverse root semantics is implemented in DWRank version. Secondly, the intended type semantics can be applied to any of the baseline ranking models to improve its performance, thus, in the DWRank+Filter version we only evaluate the effectiveness of the intended type semantics filter. The effectiveness of the framework is measured in terms of its Precision (P), Mean Average Precision (MAP), Discounted Cumulative Gain (DCG) and Normalised Discounted Cumulative Gain (NDCG).

\subsection{Experimental Results}

We next present our findings.

Effectiveness of DWRank. In the first set of experiments, we evaluated the effectiveness of DWRank in comparison with the eight baseline ranking models. We ran the ten sample queries on the ontology collection and retrieved the top- $k$ results according to the proposed ranking model. We recorded the P@10, the MAP@10, the DCG@10 and the NDCG@10. The effectiveness measure results of the DWRank are shown in Table 3 , where column header corresponds to benchmark query terms and row header corresponds to evaluation metrics.

Table 3. DWRank Effectiveness

\begin{tabular}{|l||l|l|l|l|l|l|l|l|l|l|}
\hline & Person & Name & Event & Title & Loc. & Addr. & Music & Org. & Author & Time \\
\hline P@10 & 0.9 & 0.7 & 1 & 0.7 & 0.7 & 0.8 & 0.7 & 0.9 & 0.8 & 0.8 \\
\hline MAP@10 & 0.98 & 0.82 & 1 & 0.88 & 0.86 & 0.94 & 0.80 & 0.85 & 0.78 & 0.74 \\
\hline DCG@10 & 37.58 & 19.11 & 35.12 & 12.45 & 24.88 & 23.53 & 14.82 & 33.70 & 18.24 & 22.53 \\
\hline NDCG@10 & 0.51 & 0.41 & 0.51 & 0.26 & 0.60 & 0.59 & 0.4 & 0.53 & 0.48 & 0.49 \\
\hline
\end{tabular}

Next, we compared our results with the baseline for the same dataset with the sample queries. The results are shown in Fig. 4. Each graph here presents an effectiveness measure of a ranking model for all ten queries, where the $\mathrm{x}$-axis 


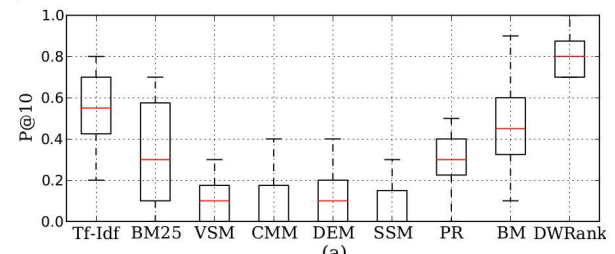

(a)

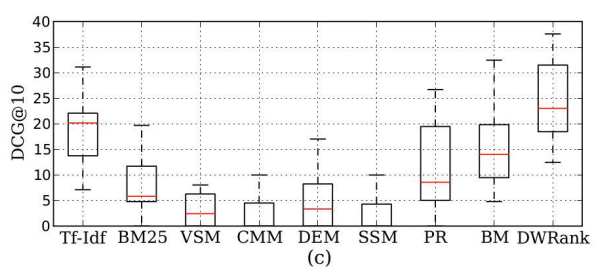

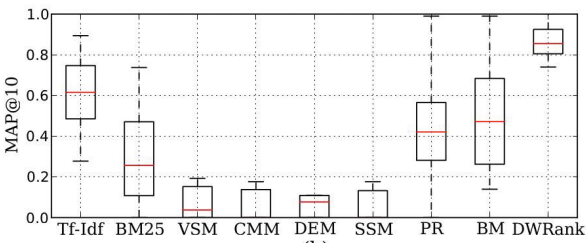

(b)

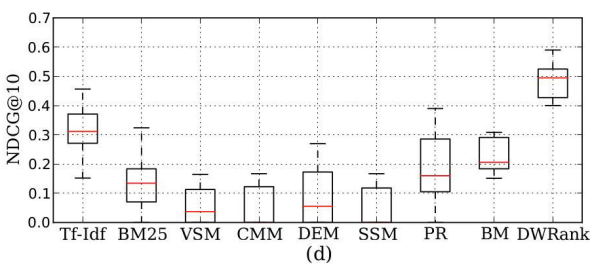

(d)

Fig. 4. Effectiveness of Ranking Model

is the ranking model and the y-axis is the unit of measure. Each box on a graph presents the range of effectiveness measure for 10 sample queries according to the gold standard. Fig. 4 shows the maximum, minimum and average performance of DWRank in comparison to the performance of the baseline ranking models for each of the ten queries. The graph shows that DWRank performs better than the best performing ranking algorithm for most queries. For the address query, the P@10 and MAP@10 for DWRank is lower than the other best performing ranking model. However, the maximum average MAPQ10 for DWRank on ten queries is 0.84 that is greater than the average of Tf-Idf, the best baseline ranking models, (i.e., 0.55). The box plot also shows that P@10 and MAP@10 of DWRank ranges from $0.7 \sim 1.0$ that means the performance of DWRank is more stable on the ontology collection for the sample queries than the baseline ranking models.

Similarly, the DCG@10 values in Fig.4(c) and NDCG@10 values in Fig. 4(d) for the ranking models show that DWRank is more effective than the baseline models. The maximum and minimum measures are closer to the Betweenness Measure (BM) and the Tf-Idf model, however, the average performance of DWRank is much higher than the average performance of the BM and Tf-Idf models.

Fig. 5] compares the MAPQ10 (resp. NDCGQ10) for DWRank on all ten queries with the maximum MAPQ10 (resp. NDCG@10) achieved with any of the baseline ranking model on the sample queries. The result shows that DWRank performs best for MAP@10 (resp. NDCG@10) for all but one query. The experiment confirms our claim about the stable performance of the DWRank algorithm.

Effectiveness of DWRank+Filter. For the evaluation of the filter performance, we ran the ten sample queries of the benchmark collection with the DWRank model extended with the filter proposed earlier, i.e. intended type semantics. Fig. [6 shows the effectiveness of DWRank compared to DWRank+Filter. 

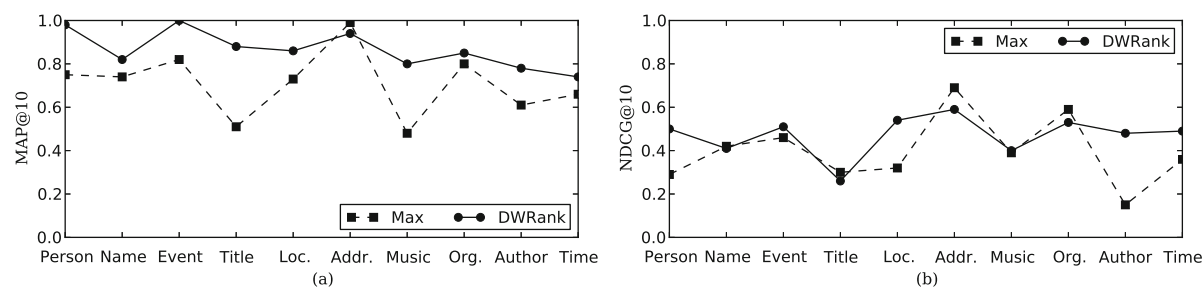

Fig. 5. $M A P @ 10$ and $n D C G @ 10$ for DWRank in comparison with the best value for any ranking model on sample queries

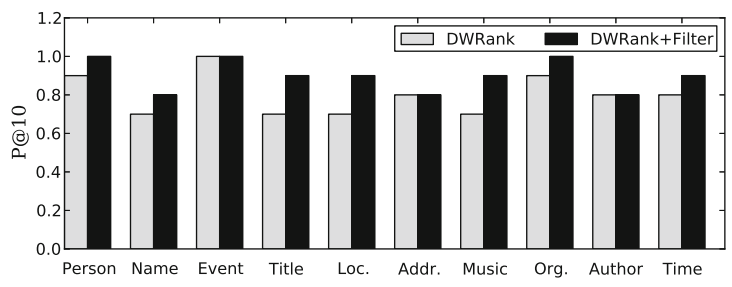

Fig. 6. Filter Effectiveness

The average P@10 increased from 0.8 to 0.9 , i.e. a $12 \%$ increase in the effectiveness of the results.

From the evaluation it is obvious that the filter improves the overall performance of our framework. A detailed analysis on the precision and recall of the intended type semantics filter is omitted from this paper for brevity. However, some True positive (TP), False positive(FP), True negative (TN) and False negative (FN) examples regarding our current implementation of the intended type semantic filter are shown in Table 4 . We analyse the top-10 results of DWRank without intended type semantic filter and then with the filter. For each query if there are TN, FN, FP examples we selected them or otherwise a random TP example.

\section{Related Work}

The Linked Open Vocabularies (LOV) search engine5, initiated in March 2011, is to the best of our knowledge, the only purpose-built ontology search engine available on the Web. It uses a ranking algorithm based on the term popularity in Linked Open Data (LOD) and in the LOV ecosystem [21].

There are also some ontology libraries available that facilitate the locating and retrieving of potentially relevant ontology resources 13. Some of these libraries are domain-specific such as the Open Biological and Biomedical Ontologies library6 or the BioPortal [14], whereas others are more general such as

\footnotetext{
5 http://lov.okfn.org

6 http://www.obofoundry.org/
} 
Table 4. Intended Type Semantic Filter Performance in Relationship-based top-k Concept Retrieval Framework

\begin{tabular}{|c|c|c|c|}
\hline Query term & Label of concept & $\begin{array}{c}\text { Human } \\
\text { Judgement }\end{array}$ & $\begin{array}{c}\text { Intended Type } \\
\text { Filter Judgement }\end{array}$ \\
\hline \hline person & personal communication model & $\times$ & $\times$ \\
\hline name & gene name & $\times$ & $\times$ \\
\hline event & academic event & $\checkmark$ & $\checkmark$ \\
\hline title & spectrum title & $\times$ & $\times$ \\
\hline location & hematopoiesis location trait & $\times$ & $\times$ \\
\hline address & E45_address & $\checkmark$ & $\times$ \\
\hline music & sound and music computing & $\times$ & $\times$ \\
\hline organization & 3D structural organization datrum & $\times$ & $\times$ \\
\hline author & author list & $\checkmark$ & $\times$ \\
\hline time & time series observation & $\times$ & $\checkmark$ \\
\hline
\end{tabular}

OntoSearch [19] or the TONES Ontology Repository 7. However, as discussed by Noy \& d'Aquin [13] only few libraries support a keyword search, only one (Cupboard [4]) supports a ranking of ontologies based on a keyword query using an information retrieval algorithm (i.e. tf-idf), and none support the ranking of resources within these ontologies.

Semantic Search engines such as Swoogle [6] (which was initially developed to rank ontologies only), Sindice.com [20, Watson [5], or Yars2 [10] do allow a search of ontology resources through a keyword query. The ranking in these search engines follows traditional link-based ranking methods [12, in particular adapted versions of the PageRank algorithm [15], where links from one source of information to another are regarded as a 'positive vote' from the former to the latter. Often, these ranking schemes also take the provenance graph of the data into account [1]. AKTiveRank [1], ranks ontologies based on how well they cover specified search terms. Falcon [3] is a popularity-based scheme to rank concepts and ontologies. Other strategies, mainly based on methods proposed in the information retrieval community, are employed in Semantic Search [8], but what all these methods have in common is that they are targeted to rank instances, but do not work well for ranking concepts and properties in ontologies [7]. Another related approach is presented in [22] that identifies the most important concepts and relationships from a given ontology. However, the approach does not support ranking concept that belong to multiple ontologies.

\section{Conclusion and Future Work}

In this paper we have presented a relationship-based top- $k$ concept retrieval and ranking framework for ontology search. The ranking model is comprised of two phases, an offline ranking and index construction phase and an online query and

\footnotetext{
7 http://owl.cs.manchester.ac.uk/repository/
} 
evaluation phase. In the offline ranking phase our DWRank algorithm computes a rank for a concept based on two features, the centrality of the concept in the ontology, and the authority of the ontology that defines the concept. The online ranking phase filters the top-k ranked list of concepts by removing redundant results and by determining the intended type of the query term and removing concept types that are not closely related to the intended query type. We evaluated our DWRank algorithm without the online query processing filters against state-of-the-art ranking models on a benchmark ontology collection and also evaluated the added performance of the proposed filters. The evaluation shows that DWRank outperforms the best performing ranking algorithm for most queries while exhibiting a more stable performance (i.e. MAP@10 of 0.84 ) than the average of the best performing ranking models of the benchmark (i.e. MAP@10 of 0.55 ). The filters proposed in the online ranking phase further increased the average $\mathrm{P} @ 10$ by $12 \%$. Although our algorithm shows significantly improved performance compared to the state-of-the-art in ontology ranking models, we believe further improvements are possible through learning the weights in computing the authority and the hub score using linear classification model. Also, in the online query processing phase we could pre-compute indices for the diverse result semantics and intended type semantics to increase the performance of the online query.

\section{References}

1. Alani, H., Brewster, C., Shadbolt, N.: Ranking Ontologies with AKTiveRank. In: Proceedings of the International Semantic Web Conference (ISWC), pp. 5-9 (2006)

2. Butt, A.S., Haller, A., Xie, L.: Ontology search: An empirical evaluation. In: Proceedings of the International Semantic Web Conference, Riva del Gara, Italy, pp. 130-147 (2014)

3. Cheng, G., Ge, W., Qu, Y.: Falcons: searching and browsing entities on the semantic web. In: Proceedings of the 17th International Conference on World Wide Web, pp. 1101-1102. ACM (2008)

4. d'Aquin, M., Lewen, H.: Cupboard - A Place to Expose Your Ontologies to Applications and the Community. In: Proceedings of the 6th European Semantic Web Conference, Berlin, Heidelberg, pp. 913-918 (2009)

5. d'Aquin, M., Motta, E.: Watson, More Than a Semantic Web Search Engine. Semantic Web 2(1), 55-63 (2011)

6. Ding, L., Finin, T., Joshi, A., Pan, R., Cost, R.S., Peng, Y., Reddivari, P., Doshi, V., Sachs, J.: Swoogle: A Search and Metadata Engine for the Semantic Web. In: Proceedings of the 13th ACM International Conference on Information and Knowledge Management, pp. 652-659. ACM, New York (2004)

7. Ding, L., Pan, R., Finin, T.W., Joshi, A., Peng, Y., Kolari, P.: Finding and ranking knowledge on the semantic web. In: Gil, Y., Motta, E., Benjamins, V.R., Musen, M.A. (eds.) ISWC 2005. LNCS, vol. 3729, pp. 156-170. Springer, Heidelberg (2005)

8. Fernandez, M., Lopez, V., Sabou, M., Uren, V., Vallet, D., Motta, E., Castells, P.: Semantic Search Meets the Web. In: Proceedings of the 2008 IEEE International Conference on Semantic Computing, Washington, DC, USA, pp. 253-260 (2008)

9. Fogaras, D.: Where to start browsing the web? In: Innovative Internet Community Systems, pp. 65-79 (2003) 
10. Harth, A., Umbrich, J., Hogan, A., Decker, S.: YARS2: A Federated Repository for Querying Graph Structured Data from the Web. In: Aberer, K., et al. (eds.) ASWC 2007 and ISWC 2007. LNCS, vol. 4825, pp. 211-224. Springer, Heidelberg (2007)

11. Hogan, A., Harth, A., Decker, S.: Reconrank: A scalable ranking method for semantic web data with context. In: Proceedings of the 2nd Workshop on Scalable Semantic Web Knowledge Base Systems (2006)

12. Hogan, A., Harth, A., Umbrich, J., Kinsella, S., Polleres, A., Decker, S.: Searching and browsing Linked Data with SWSE: The Semantic Web Search Engine. Web Semantics: Science, Services and Agents on the World Wide Web 9(4), 365-401 (2011)

13. Noy, N.F., d'Aquin, M.: Where to Publish and Find Ontologies? A Survey of Ontology Libraries. Web Semantics: Science, Services and Agents on the World Wide Web 11(0) (2012)

14. Noy, N.F., Shah, N.H., Whetzel, P.L., Dai, B., Dorf, M., Griffith, N., Jonquet, C., Rubin, D.L., Storey, M.-A., Chute, C.G., Musen, M.A.: BioPortal: ontologies and integrated data resources at the click of a mouse. Nucleic Acids Research (2009)

15. Page, L., Brin, S., Motwani, R., Winograd, T.: The PageRank citation ranking: Bringing order to the Web. In: Proceedings of the 7th International World Wide Web Conference, Brisbane, Australia, pp. 161-172 (1998)

16. Robertson, S.E., Walker, S., Jones, S., Hancock-Beaulieu, M.M., Gatford, M.: Okapi at trec-3. NIST SPECIAL PUBLICATION SP, 109-109 (1995)

17. Salton, G., Buckley, C.: Term-weighting approaches in automatic text retrieval. Information Processing \& Management 24(5), 513-523 (1988)

18. Salton, G., Wong, A., Yang, C.-S.: A vector space model for automatic indexing. Communications of the ACM 18(11), 613-620 (1975)

19. Thomas, E., Pan, J.Z., Sleeman, D.: Ontosearch2: Searching ontologies semantically. In: Proceedings of the OWLED 2007 Workshop on OWL: Experiences and Directions. CEUR Workshop Proceedings, vol. 258 (2007)

20. Tummarello, G., Delbru, R., Oren, E.: Sindice.com: Weaving the Open Linked Data. In: Aberer, K., et al. (eds.) ASWC 2007 and ISWC 2007. LNCS, vol. 4825, pp. 552-565. Springer, Heidelberg (2007)

21. Vandenbussche, P.-Y., Vatant, B.: Linked Open Vocabularies. ERCIM News 96, 21-22 (2014)

22. Wu, G., Li, J., Feng, L., Wang, K.-H.: Identifying potentially important concepts and relations in an ontology. In: Sheth, A.P., Staab, S., Dean, M., Paolucci, M., Maynard, D., Finin, T., Thirunarayan, K. (eds.) ISWC 2008. LNCS, vol. 5318, pp. 33-49. Springer, Heidelberg (2008) 